\title{
An integrated bioinformatical analysis of miR-19a target genes in multiple myeloma
}

\author{
HONGYAN LV*, XIANDA WU*, GUIRU MA, LIXIA SUN, JIANBO MENG, \\ XIAONING SONG and JINQIAO ZHANG \\ Department of Hematology, Third Hospital of Hebei Medical University, Shijiazhuang, Hebei 050051, P.R. China \\ Received September 12, 2016; Accepted May 19, 2017
}

DOI: $10.3892 /$ etm.2017.5173

\begin{abstract}
MicroRNA (miR)-19a, as an oncomiR, has been studied in several types of cancer; however, its role in the development and progression of multiple myeloma (MM) remains unclear. The present study used a bioinformatics approach to investigate the involvement of miR-19a in MM. miR-19a targets were predicted using target prediction programs, followed by screening for differentially expressed genes in MM. The function of these genes was then annotated using gene ontology term enrichment, signaling pathway enrichment and protein-protein interaction (PPI) analysis. In addition, natural language processing (NLP) was performed to identify genes associated with MM. A total of 715 putative targets of miR-19a were identified in the present study, of which 40 were experimentally validated. A total of 121 genes were identified to be differentially expressed in MM, including 80 upregulated genes and 41 downregulated genes. Among the differentially expressed genes, ras homolog family member B, clathrin heavy chain, prosaposin and protein phosphatase 6 regulatory subunit 2 were predicted target genes of miR-19a. The results of NLP revealed that 2 of the differentially expressed genes, Y-box binding protein 1 and TP53 regulated inhibitor of apoptosis 1, were reported to be associated with MM. In addition, 41 target genes of miR-19a were identified to be associated with the development and progression of MM. These results may aid in understanding the molecular mechanisms of miR-19a in the development and progression of MM. In addition, the results of the present study indicate that targets genes of miR-19a are potential candidate biomarkers for MM.
\end{abstract}

Correspondence to: Dr Jinqiao Zhang, Department of Hematology, Third Hospital of Hebei Medical University, 139 Ziqiang Road, Shijiazhuang, Hebei 050051, P.R. China

E-mail: zhjq72@163.com

*Contributed equally

Key words: multiple myeloma, miR-19a, systematic analysis, gene expression

\section{Introduction}

Multiple myeloma (MM) is a B-cell malignancy characterized by the aberrant clonal expansion of plasma cells (PCs) within the bone marrow, and, as a consequence, osteolytic bone destruction with hypercalcemia, anemia, immunosuppression and end organ damage frequently occurs (1). Recent advances in molecular and genetic research into MM have led to the discovery that although MM is defined histologically as a single entity, it encompasses a wide range of genomic abnormalities, including numerical and structural chromosomal abnormalities, gene mutations and epigenetic alterations (2-4), which differ in their molecular pathogenesis and prognostic significance (5).

MicroRNAs (miRNAs/miRs) are small non-coding single-stranded RNAs of 22 nucleotides in length, which control gene expression at a post-transcriptional level by degrading or repressing target mRNAs, resulting in translational repression or mRNA degradation. miRNAs serve roles in essential biological processes, including cellular growth, differentiation and proliferation. In addition, miRNAs regulate the expression of $>30 \%$ of protein-coding genes, and $>50 \%$ of miRNA target genes are located in cancer-associated genomic regions, suggesting that miRNAs serve an important role in the pathogenesis of human cancer $(6,7)$. It is well known that the dysregulation of miRNAs is associated with the pathogenesis of cancer, and that miRNA expression profiles have prognostic implications in numerous types of cancer. Thus, inhibiting specific miRNAs is a therapeutic strategy for the treatment of cancer (8).

Numerous previous studies have detected miRNA expression in MM via microarray profiling and reverse transcription-quantitative polymerase chain reaction analysis (9-11), with results suggesting that miRNAs serve an important role in the molecular pathogenesis, progression and prognosis of MM. Lionetti et al (9) evaluated the influence of allelic imbalances on miRNA expression in MM, and identified that differential miRNA expression patterns were associated with the cytogenetic abnormalities in MM, particularly with immunoglobulin heavy locus translocations. Furthermore, Wu et al (10) was able to develop an 'outcome classifier' in patients newly diagnosed with myeloma based on their expression of specific miRNAs.

The miR-17-92 cluster, located in an intron of miR-17-92a-1 clusterhost gene on chromosome $13 \mathrm{q} 31.3$, was originally reported 
to be implicated in B-cell neoplasms, including MM (11). Later, the miR-17-92 cluster was identified as an oncomiR due to its oncogenic activity in several types of cancer (12). Mendell (11) identified that the deletion of miR-17-92 inhibited B-cell proliferation and development, whereas its overexpression induced B-cell hyperproliferation and autoimmune diseases. Another study revealed that members of the miR-17-92 cluster, particularly miR-19a and b, were upregulated in MM, but not in healthy cases or monoclonal gammopathy of undetermined significance (MGUS), suggesting a potential role of the cluster in the progression from MGUS to MM, likely representing MM-specific genetic changes (2).

miR-19a, a key component of miR-17-92 cluster, has been directly implicated in myeloma pathogenesis $(13,14)$. In addition, miR-19a has been demonstrated to be upregulated in patients with MM, and in MM cell lines compared with normal plasma cells $(13,15)$. Furthermore, miR-19a was more highly expressed in patients with MM with 13q14 deletions compared with those without these deletions $(5,16,17)$. Additionally, miR-19a antagonists have been revealed to suppress MM tumor growth in nude mice (14). miR-19a can modulate the expression of proteins that are essential in myeloma pathogenesis, including suppressors of cytokine signaling (SOCS), a gene that is frequently silenced in MM, releasing inhibition of interleukin 6 and leading to pro-growth signaling (14). These results highlight the contribution of miR-19a to the pathogenesis of $\mathrm{MM}$ and its potential application as a molecular biomarker for MM.

Although the function of miR-19a has been relatively well studied, its exact role in the development and progression of MM remains unclear. Systematic analyses of miR-19a-associated malignant cell behavior is required. Since miR-19a's function is mediated through its target genes, the exploration of its target genes is also essential. The inverse correlation between miRNA-mRNA interactions may aid in the identification of target genes regulated by miR-19a in the pathogenesis of MM. Combined with the MM gene expression profiling data generated by high-throughput technology in a previous study (18), the present study performed a systematic analysis of miR-19a predicted target genes associated with the carcinogenesis, prognosis and chemoresistance of MM in order to further investigate the potential involvement of miR-19a in MM.

\section{Materials and methods}

Prediction of miRNA target genes. miR-19a target prediction was performed using the online tool miRWalk (http://www.umm. uni-heidelberg.de/apps/zmf/mirwalk) (19) with a combination of three currently available independent target prediction programs, including PicTar (version 2005; http://pictar.mdc-berlin. de/cgi-bin/PicTar_vertebrate.cgi), miRanda (version 5; http://www.ebi.ac.uk/enright-srv/microcosm/htdocs/targets/v5) and TargetScan (version 5.1; http://www.targetscan.org). Only the targets genes that were confirmed by all of the above prediction programs or experimentally validated according to the miRWalk database were considered putative targets of miR-19a.

Identifying gene expression profiles. The Gene Expression Omnibus database (GEO, http://www.ncbi.nlm.nih.gov/geo), a public repository for high-throughput gene expression datasets, was searched for MM gene expression profiling studies. Expression profiling studies of peripheral blood mononuclear cells from patients with MM were obtained from previous studies (20-22).

Differential analysis of genes in MM. The raw gene expression data of each study was downloaded from the GEO database, and preprocessed for background correction and Z-score normalization. The Bioconductor limma package (version 1.9.6) in $\mathrm{R}$ (23) was used to perform differential analysis of genes between MM and controls using a two-tailed Student's t-test. The p-value of individual microarray studies were combined using Fisher's exact test. Differently expressed genes with a false discovery rate (FDR) $<0.01$ were selected.

Functional classification. GeneCodis (http://genecodis.cnb. csic.es/) was used to perform Gene Ontology (GO) term and Kyoto Encyclopedia of Genes and Genomes (KEGG) pathway enrichment analysis, in order to annotate the function of the selected genes. The functional GO terms were classified into three groups, biological processes, cellular components and molecular functions. Genes were mapped to corresponding signaling pathways according to KEGG signaling pathway database, and the enrichment FDR was calculated for each pathway, and the criteria of FDR $<0.05$ was used as the threshold for significance.

Protein-protein interaction (PPI) network analysis. To explore the function of genes at the protein level, PPI analysis was performed as previously described (24). The Biological General Repository for Interaction Datasets (BioGRID; http://thebiogrid.org) was used to construct the PPI network. BIOGRID is an online interaction repository, confirmed by existing high-throughput experiments. Cytoscape version 3.3.0 software was used to display the PPI network, as previously described (25). In the PPI network produced, nodes indicate proteins and edges indicate interactions between these nodes. The nodes that contain the most connectivity degrees are defined as significant hub proteins.

Natural language processing (NLP) analysis of MM. Document searching and formatting were performed in PubMed (http://www.ncbi.nlm.nih.gov/pubmed) using the keywords 'multiple myeloma' and 'resistance or prognosis or carcinogenesis or tumorigenesis'. All of the genes and proteins associated with these keywords were extracted, followed by gene mention tagging using A Biomedical Named Entity Recognizer software, version 1.5 (http://pages.cs.wisc.edu/ bsettles/abner). For the conditions, multiple genes were described in a word, such as 'STAT3/5 gene', and these were translate manually to 'STAT3 gene', and 'STAT5 gene'. Gene names were normalized based on the Entrez database (https://www.ncbi.nlm.nih .gov/gene). Gene names were normalized based on the Entrez database (https://www.ncbi.nlm.nih.gov/gene).

The frequency of the occurrence of each gene was calculated. The higher the frequency of the gene, the greater the likelihood of the association between MM and the gene. The total number of studies in PubMed database was recorded as ' $N$ '. The frequency of the genes and diseases associated 
with these in the PubMed database were denoted as ' $m$ ' and ' $n$ ', respectively. It was hypothesized that subtracting the disease co-occurrent from the actual frequency of the gene would equal ' $\mathrm{k}$ '. Then, by using hypergeometric distribution, the probability of a frequency greater than $\mathrm{k}$ co-citation at completely random conditions was calculated as follows:

$$
\begin{aligned}
& p=1-\sum_{i=0}^{k-1} p(i \mid n, m, N) \\
& p(i \mid n, m, N)=\frac{n !(N-n) ! m !(N-m) !}{(n-i) ! i !(n-m) !(N-n-m+i) ! N !}
\end{aligned}
$$

\section{Results}

Predicted target genes of miR-19a. Target genes of miR-19a were predicted using three target prediction programs. A total of 715 putative targets of miR-19a were identified using these three programs, among which 40 were experimentally validated in miRWalk (data not shown).

Differentially expressed genes in MM. Following searching the GEO database, three gene expression profiling studies of MM were collected (GSE23832, GSE21942 and GSE17048; Table I). The raw data was downloaded and processed, and 121 genes were identified to be differentially expressed in MM with an FDR $<0.01$, including 80 upregulated genes and 41 downregulated genes (Fig. 1). The top 10 most significantly upregulated or downregulated genes are listed in Table II. Interestingly, four putative targets of miR-19a, ras homolog family member B (RHOB), clathrin heavy chain (CLTC), prosaposin (PSAP) and protein phosphatase 6 regulatory subunit 2 (PPP6R2), were identified to be differentially expressed.

Functional classification of differentially expressed genes. For the differentially expressed genes in MM, GO term enrichment analysis was performed. For biological processes, 38 GO terms were significantly enriched, and the most significantly enriched

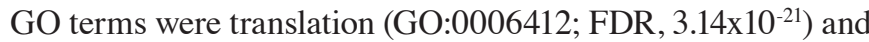
viral transcription (GO:0019083; FDR, 3.59 $\times 10^{-18}$ ) (Fig. 2A). For cellular components, 29 GO terms were significantly enriched, and the most significantly enriched GO terms were ribosome (GO:0005840; FDR, 5.12 $\times 10^{-15}$ ) and cytosolic small ribosomal subunit (GO:0022627; FDR, 2.60×10-11) (Fig. 2B). For molecular functions, $9 \mathrm{GO}$ terms were significantly enriched, and the most significantly enriched GO terms were structural constituent of ribosome (GO:0003735; FDR, 1.40×10 ${ }^{-20}$ ) and protein binding (GO:0005515; FDR, 1.82×10-10) (Fig. 2C).

When performing the KEGG pathway enrichment analysis, 7 signaling pathways were significantly enriched with the criteria of FDR $<0.05$ (Table III). The most significant pathway was ribosome (FDR, 2.25 $\left.\times 10^{-16}\right)$. Furthermore, oxidative phosphorylation (FDR, 0.0277), bacterial invasion of epithelial cells (FDR, 0.0286), lysosome (FDR, 0.0318), the Wnt signaling pathway (FDR, 0.0343), tuberculosis (FDR, 0.0385) and collecting duct acid secretion (FDR, 0.0407) were also significantly enriched.

PPI network. A PPI network including all of the differentially expressed genes identified was constructed. The PPI network produced included 2,355 nodes and 3,707 edges (Fig. 3). Highly connected proteins in the network are called hub proteins, which are the core of regulation and serve an important role in the stability of the network. The significant hub proteins were identified, including COP9 signalosome complex subunit 5 (COPS5; connectivity degree, 791), CLTC (connectivity degree, 172) and 60S ribosomal protein L11 (connectivity degree, 167).

NLP results. The abstracts of 6,795 primary studies were identified using the aforementioned search strategy and a total of $662 \mathrm{MM}$-associated genes were obtained (data not shown). Integrated analysis was performed to identify the overlap between the $715 \mathrm{miR}-19 \mathrm{a}$ target genes previously identified and the $662 \mathrm{MM}$-associated genes obtained from NLP analysis. This revealed that there were 41 overlapping genes (Table IV and Fig. 4), which were associated with the development and progression of MM, and were putative miR-19a target genes. In addition, 2 of the differentially expressed genes, Y-box binding protein 1 (YBX1) and TP53 regulated inhibitor of apoptosis 1 (TRIAP1) were identified to be associated with MM (Fig. 4).

\section{Discussion}

Several previous studies $(2,13-15)$ have demonstrated that miR-19a is deregulated in MM as an oncomiR, suggesting it serves an important role in MM. Considering that the biological significance of miRNA deregulation relies on the effect upon target protein-coding genes, predicted target genes of miR-19a that were associated with the carcinogenesis, prognosis and chemoresistance of MM were systematically analyzed in the present study in order to further investigate the potential involvement of miR-19a in MM. Strategies to determine miRNA targets include bioinformatical prediction and experimental assays. The present study utilized three common computational algorithms, miRanda, PicTar and TargetScan, to identify 715 putative target genes of miR-19a, among which 40 were experimentally validated in miRWalk. In addition, NLP analysis was performed in the current study, which identified 662 MM-associated genes. Then, integrated analysis revealed 41 predicted target genes of miR-19a that were associated with the development and progression of MM, including Kirsten rat sarcoma viral oncogene homolog (KRAS), SOCS and CCND1. Several of these MM-associated putative miR-19a targets, including SOCS and CCND1, have already been verified by miRNA functional experiments (14). Previous studies have demonstrated that oncogenic mutations of RAS occur in $30-40 \%$ of patients with MM and are rarely found in MGUS (26-28). The occurrence of RAS mutation appears independent of clinical stage, but is associated with disease progression, an aggressive phenotype, resistance to therapy and poor patient survival $(26,27,29)$. Steinbrunn et al (27) also reported that the ectopic overexpression of oncogenic RAS induces MM cell proliferation and lowers drug efficacy.

Given that the altered expression of miR-19a in MM would cause changes in target gene expression, differentially expressed genes between MM and normal controls were assessed in current study using gene expression data. This revealed that 121 genes were differentially expressed in MM, 
Table I. Characteristics of the three gene expression profiling datasets for multiple myeloma downloaded for integrated analysis.

\begin{tabular}{|c|c|c|c|c|}
\hline $\begin{array}{l}\text { Author, } \\
\text { year }\end{array}$ & $\begin{array}{c}\text { GEO } \\
\text { dataset ID }\end{array}$ & Platform for detection & $\begin{array}{l}\text { Samples } \\
(\mathrm{N}: \mathrm{M})\end{array}$ & (Refs.) \\
\hline $\begin{array}{l}\text { Zhang et al, } \\
2011\end{array}$ & GSE23832 & $\begin{array}{l}\text { GPL6244 [HuGene-1_0-st] Affymetrix Human } \\
\text { Gene 1.0 ST Array [transcript (gene) version] }\end{array}$ & $4: 8$ & (20) \\
\hline $\begin{array}{l}\text { Kemppinen et al, } \\
2011\end{array}$ & GSE21942 & $\begin{array}{l}\text { GPL570 [HG-U133_Plus_2] Affymetrix Human } \\
\text { Genome U133 Plus 2.0 Array }\end{array}$ & $15: 12$ & (21) \\
\hline $\begin{array}{l}\text { Gandhi et al, } \\
2010\end{array}$ & GSE17048 & $\begin{array}{l}\text { GPL6947 Illumina HumanHT-12 V3.0 expression } \\
\text { beadchip }\end{array}$ & $45: 99$ & (22) \\
\hline
\end{tabular}

GEO, Gene Expression Omnibus; N:M, normal:multiple myeloma.

Table II. Top 10 significantly upregulated and downregulated DEGs identified in multiple myeloma. All gene ID's were taken from the Entrez database on NCBI.

A, Upregulated DEGs

\begin{tabular}{|c|c|c|c|}
\hline Entrez gene ID & Abbreviation & Name & FDR \\
\hline 84265 & POLR3GL & $\begin{array}{l}\text { Polymerase (RNA) III (DNA directed) polypeptide G } \\
(32 \mathrm{kD}) \text {-like }\end{array}$ & 0.0001793 \\
\hline 8364 & HIST1H4C & Histone cluster $1, \mathrm{H} 4 \mathrm{c}$ & 0.0002646 \\
\hline 6170 & RPL39 & Ribosomal protein L39 & 0.0013368 \\
\hline 5880 & RAC2 & $\begin{array}{l}\text { Ras-related C3 botulinum toxin substrate } 2 \text { (rho } \\
\text { family, small GTP binding protein Rac2) }\end{array}$ & 0.0013368 \\
\hline 29080 & CCDC59 & Coiled-coil domain containing 59 & 0.0013368 \\
\hline 521 & ATP5I & $\begin{array}{l}\text { ATP synthase, } \mathrm{H}+\text { transporting, mitochondrial Fo } \\
\text { complex, subunit E }\end{array}$ & 0.0013773 \\
\hline 9991 & PTBP3 & Polypyrimidine tract binding protein 3 & 0.001458 \\
\hline 79023 & NUP37 & Nucleoporin $37 \mathrm{kDa}$ & 0.001458 \\
\hline 64801 & ARV1 & ARV1 homolog, fatty acid homeostasis modulator & 0.001458 \\
\hline 3700 & ITIH4 & $\begin{array}{l}\text { Inter- } \alpha \text {-trypsin inhibitor heavy chain family, } \\
\text { member } 4\end{array}$ & 0.001458 \\
\hline
\end{tabular}

B, Downregulated DEGs

\begin{tabular}{lllr}
\hline Entrez gene ID & Abbreviation & \multicolumn{1}{c}{ Name } & FDR \\
\hline 387 & RHOA & Ras homolog family member A & 0.0004738 \\
23256 & SCFD1 & Sec1 family domain containing 1 & 0.0013368 \\
92241 & RCSD1 & RCSD domain containing 1 & 0.0014580 \\
126364 & LRRC25 & Leucine rich repeat containing 25 & 0.0017804 \\
5226 & PGD & Phosphogluconate dehydrogenase & 0.0026507 \\
129531 & MITD1 & MIT, microtubule interacting and transport, domain & 0.0027447 \\
10023 & & containing 1 & 0.0027447 \\
8883 & FRAT1 & Frequently rearranged in advanced T-cell lymphomas 1 & 0.0028332 \\
81631 & NAE1 & NEDD8 activating enzyme E1 subunit 1 & 0.0028998 \\
4904 & MAP1LC3B & Microtubule-associated protein 1 light chain 3 $\beta$ & 0.0028998 \\
& YBX1 & Y box binding protein 1 &
\end{tabular}

DEGs, differentially expressed genes; FDR, false discovery rate. Entrez database: https://www.ncbi.nlm.nih.gov/gene. 
Table III. Significantly enriched KEGG signaling pathways of the differentially expressed genes identified in multiple myeloma.

\begin{tabular}{|c|c|c|c|c|}
\hline $\begin{array}{l}\text { KEGG } \\
\text { ID }\end{array}$ & $\begin{array}{l}\text { KEGG } \\
\text { term }\end{array}$ & $\begin{array}{l}\text { No. of } \\
\text { enriched genes }\end{array}$ & FDR & Genes \\
\hline hsa03010 & Ribosome & 13 & $2.25 \times 10^{-16}$ & $\begin{array}{l}\text { RPS15A, RPS27A, RPS25, RPS13, RPL26,RPL39, } \\
\text { RPL21, RPS29, RPS14, RPS27, RPL11, RPL27, } \\
\text { RPS10 }\end{array}$ \\
\hline hsa00190 & $\begin{array}{l}\text { Oxidative } \\
\text { phosphorylation }\end{array}$ & 4 & $2.77 \times 10^{-02}$ & NDUFS4, ATP6V1A, ATP5I, ATP6V0C \\
\hline hsa05100 & $\begin{array}{l}\text { Bacterial invasion of } \\
\text { epithelial cells }\end{array}$ & 3 & $2.86 \times 10^{-02}$ & CLTC, RHOA, CRKL \\
\hline hsa04142 & Lysosome & 4 & $3.18 \times 10^{-02}$ & CLTC, PSAP, ATP6V0C, LAMP2 \\
\hline hsa04310 & $\begin{array}{l}\text { Wnt signaling } \\
\text { pathway }\end{array}$ & 4 & $3.43 \times 10^{-02}$ & SIAH1, RAC2, RHOA, FRAT1 \\
\hline hsa05152 & Tuberculosis & 4 & $3.85 \times 10^{-02}$ & APAF1, RHOA, ATP6V0C, LAMP2 \\
\hline hsa04966 & $\begin{array}{l}\text { Collecting duct } \\
\text { acid secretion }\end{array}$ & 2 & $4.07 \times 10^{-02}$ & ATP6V1A, ATP6V0C \\
\hline
\end{tabular}

KEGG, Kyoto Encyclopedia of Genes and Genomes; FDR, false discovery rate.

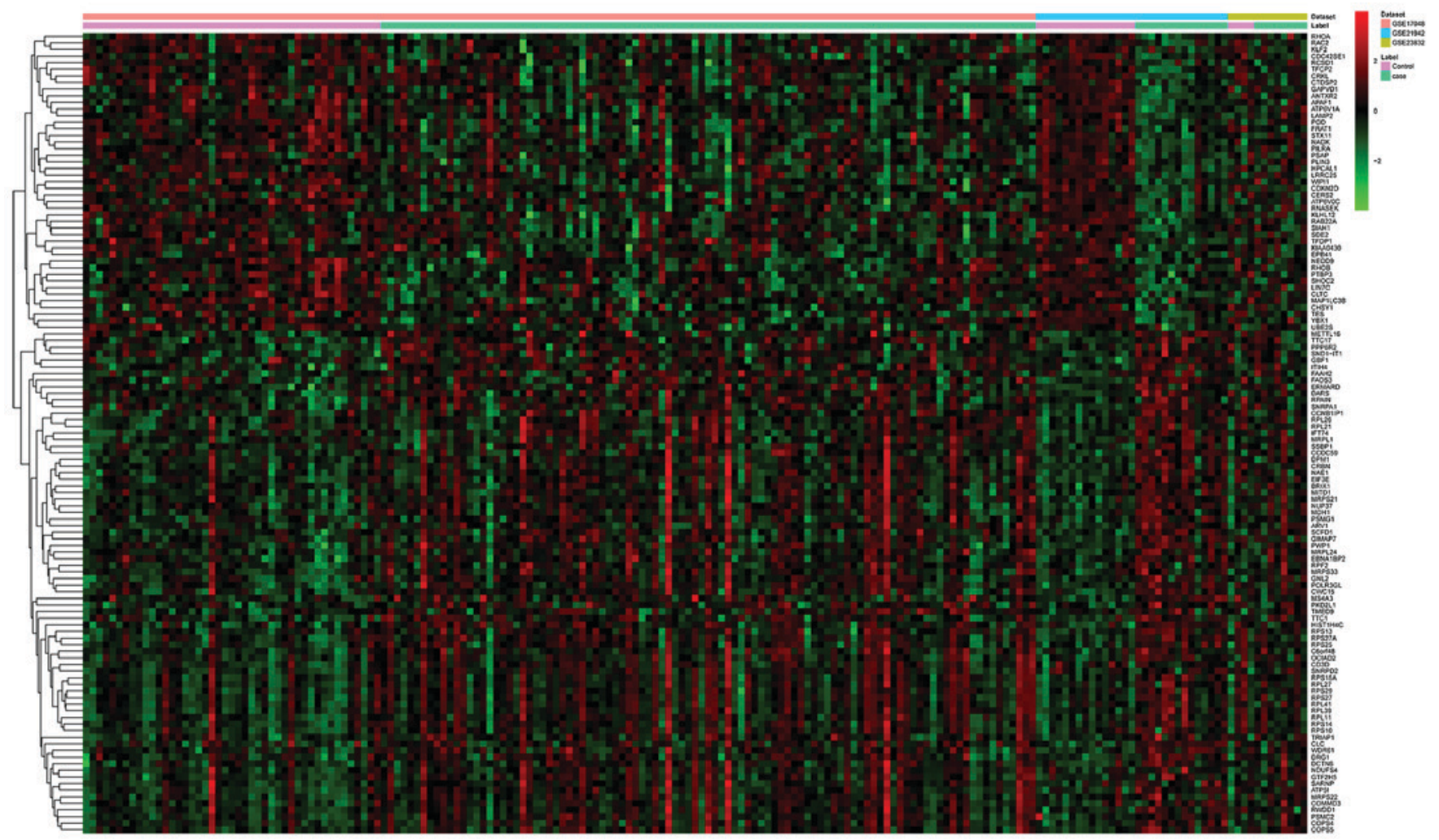

Figure 1. Heatmap of the 121 differentially expressed genes identified in multiple myeloma from the three datasets.

including 80 upregulated genes and 41 downregulated genes. In addition, 2 of the differentially expressed genes, YBX1 and TRIAP1, were identified to be associated with MM in the present study. YBX1, a member of the cold-shock domain protein superfamily, is involved in a wide range of cellular functions, including DNA transcription, replication and repair, and environmental stress and chromatin remodeling, in addition to pre-mRNA splicing (30). Chatterjee et al (31) demonstrated that YBX1 was overexpressed in immature and anaplastic MM cells, but not expressed in normal PCs, MGUS PCs or the majority of MM specimens, suggesting it serves a role in dedifferentiation as part of the malignant transformation process. Furthermore, other studies have reported that the aberrant expression of YBX1 is associated with tumorigenesis, and cancer cell proliferation, survival and drug resistance $(32,33)$.

Interestingly, four putative targets of miR-19a, RHOB, CLTC, PSAP and PPP6R2, were identified to be differentially 


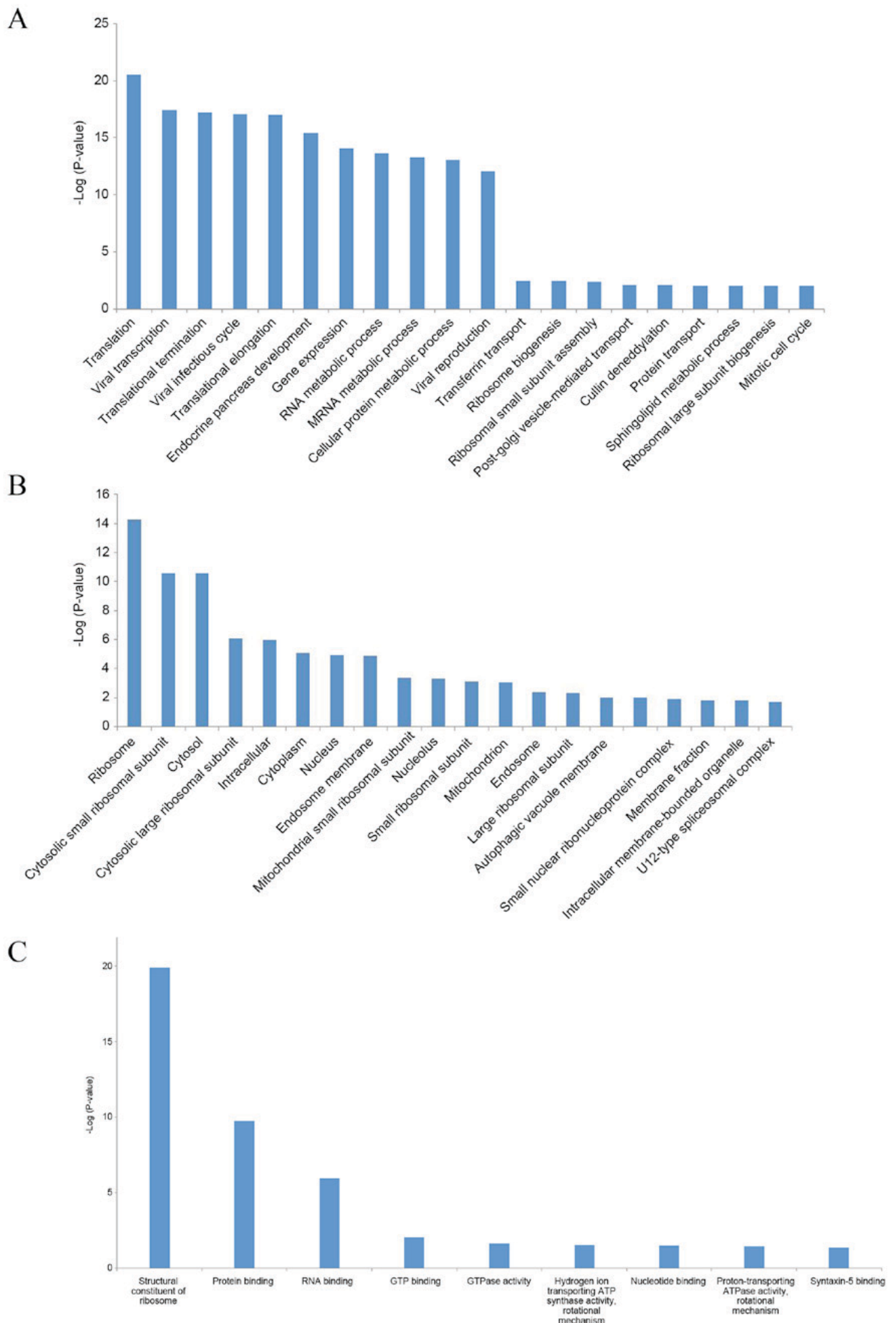

Figure 2. GO term enrichment analysis of the differentially expressed genes identified in multiple myeloma. GO analysis under the category of (A) biological processes, (B) cellular components and (C) molecular functions. GO, gene ontology.

expressed in MM in the present study. The tumor suppressor RHOB has been demonstrated to downregulated in various types of cancer $(34,35)$, which is in accord with the findings of the present study. Notably, Tan et al (36) revealed 


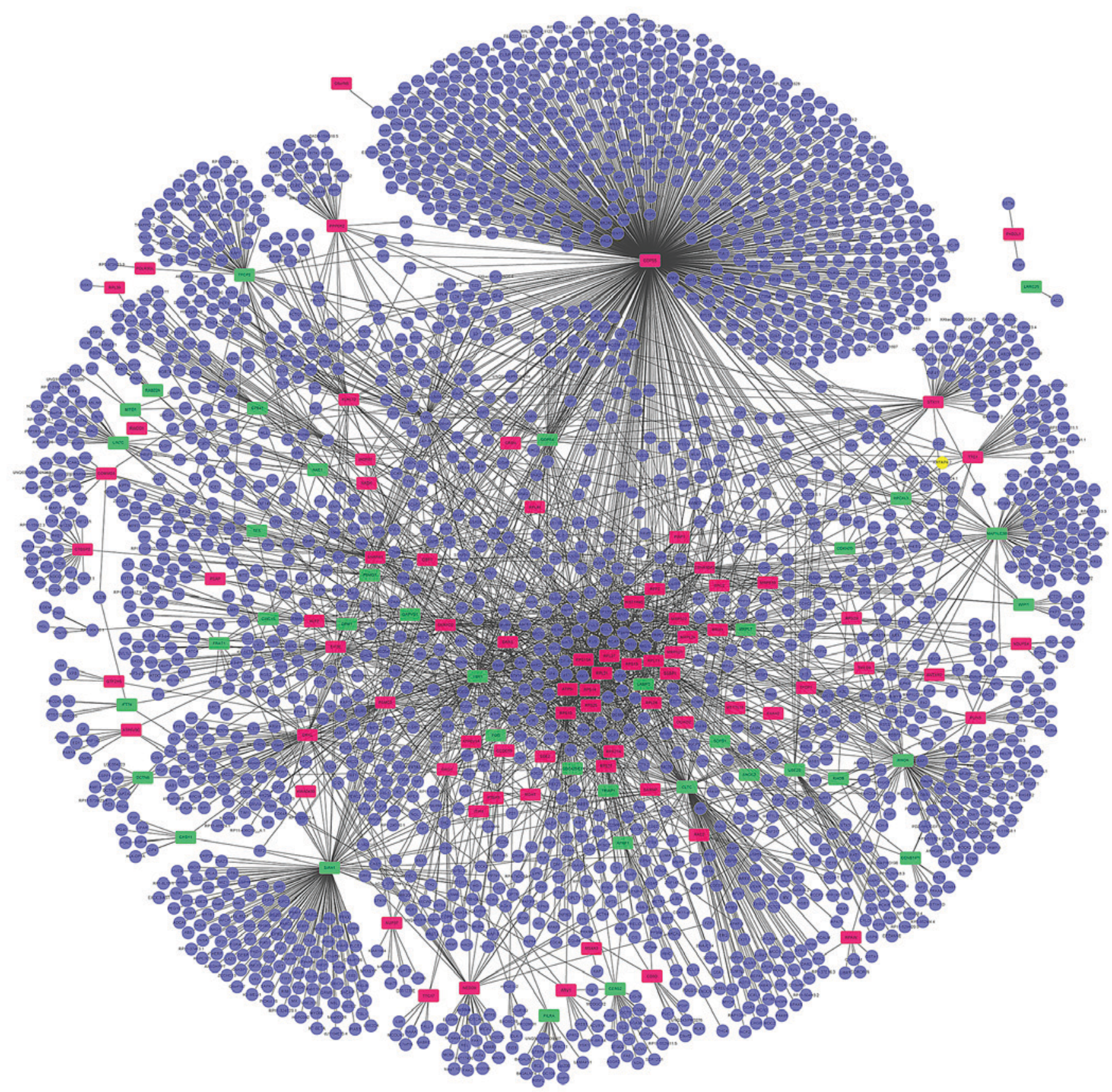

Figure 3. Protein-protein interaction analysis of the differentially expressed genes identified in multiple myeloma. Red, upregulated; green, downregulated; rectangles, differentially expressed genes; circular, genes interacting with differentially expressed genes.

that RHOB induced apoptosis, and inhibited proliferation and migration in pancreatic cancer as a direct target of miR-19a. Chromosomal and genomic analyses have revealed that the ALK receptor tyrosine kinase gene is fused to CLTC in inflammatory myofibroblastic tumors and B-cell lymphoma $(37,38)$. However, the exact role of CLTC in MM has not yet been reported. PSAP, a highly conserved glycoprotein, is overexpressed in prostate cancer and esophageal squamous cell carcinoma $(39,40)$. A similar expression trend for PSAP was observed in the present study, indicating that it may be a candidate biomarker for MM.

In the PPI network of differentially expressed genes produced in the present study, COPS5 had the highest connectivity degree, suggesting that it serves an important role in MM progression. COPS5, one of the eight subunits of the COP9 signalosome, is overexpressed in a variety of types of human cancer (41). COPS5was identified to be overexpressed in MM in the present study. A previous study demonstrated that the specific knockdown of COPS5 inhibits the proliferation of colorectal cancer cells (42), and that COPS5-transgenic mice developed a phenotype similar to that of myeloproliferative disorders (43). In addition, COPS5 is involved in Ras-mediated cell transformation by inhibiting premature senescence (44).

All of the differentially expressed genes in MM identified in the present study underwent GO term and signaling pathway enrichment analysis, in addition to PPI network construction, in order to understand their function. This revealed functions in 
Table IV. Overlapping genes that were associated with the development and progression of multiple myeloma, and were putative target genes of microRNA-19a $(n=41)$.

\begin{tabular}{|c|c|c|c|}
\hline $\begin{array}{l}\text { Gene } \\
\text { abbreviation }\end{array}$ & Gene name & Count & P-value \\
\hline CCND1 & Cyclin D1 & 78 & $<0.0001$ \\
\hline CCNA2 & Cyclin A2 & 1 & 0.2128 \\
\hline CCND2 & Cyclin D2 & 16 & $<0.0001$ \\
\hline CD69 & CD69 molecule & 1 & 0.0818 \\
\hline CTGF & Connective tissue growth factor & 1 & 0.2861 \\
\hline S1PR1 & Sphingosine-1-phosphate receptor 1 & 1 & 0.1044 \\
\hline EREG & Epiregulin & 1 & 0.0388 \\
\hline ESR1 & Estrogen receptor 1 & 3 & 0.4207 \\
\hline F3 & Coagulation factor III (thromboplastin, tissue factor) & 2 & 0.0853 \\
\hline GJA1 & Gap junction protein, $\alpha 1,43 \mathrm{kDa}$ & 1 & 0.2950 \\
\hline GRK6 & G protein-coupled receptor kinase 6 & 1 & 0.0625 \\
\hline ID2 & Inhibitor of DNA binding 2, dominant negative helix-loop-helix protein & 1 & 0.1265 \\
\hline IL6ST & Interleukin 6 signal transducer (gp130, oncostatin M receptor) & 2 & 0.0231 \\
\hline ITGA6 & Integrin, $\alpha 6$ & 1 & 0.1979 \\
\hline KIT & V-kit Hardy-Zuckerman 4 feline sarcoma viral oncogene homolog & 10 & $<0.0001$ \\
\hline KRAS & V-Ki-ras2 Kirsten rat sarcoma viral oncogene homolog & 1 & 0.5071 \\
\hline LIF & leukemia inhibitory factor (cholinergic differentiation factor) & 1 & 0.1516 \\
\hline SMAD4 & SMAD family member 4 & 1 & 0.3981 \\
\hline MDM4 & Mdm4 p53 binding protein homolog (mouse) & 1 & 0.1155 \\
\hline PTEN & Phosphatase and tensin homolog & 5 & 0.0038 \\
\hline ATXN1 & Ataxin 1 & 1 & 0.1155 \\
\hline SDC1 & Syndecan 1 & 46 & $<0.0001$ \\
\hline TGFBR2 & Transforming growth factor, $\beta$ receptor II (70/80kDa) & 1 & 0.3081 \\
\hline THBS1 & Thrombospondin 1 & 1 & 0.3418 \\
\hline KLF10 & Kruppel-like factor 10 & 1 & 0.0487 \\
\hline TNFAIP3 & Tumor necrosis factor, $\alpha$-induced protein 3 & 2 & 0.0064 \\
\hline SOCS1 & Suppressor of cytokine signaling 1 & 8 & $<0.0001$ \\
\hline SOCS3 & Suppressor of cytokine signaling 3 & 3 & 0.0022 \\
\hline HDAC4 & Histone deacetylase 4 & 1 & 0.1463 \\
\hline FOXP1 & Forkhead box P1 & 2 & 0.0016 \\
\hline MIB1 & Mindbomb homolog 1 (Drosophila) & 1 & 0.0267 \\
\hline PCDH10 & Protocadherin 10 & 1 & 0.0226 \\
\hline CYLD & Cylindromatosis (turban tumor syndrome) & 1 & 0.1007 \\
\hline PTK2B & PTK2B protein tyrosine kinase $2 \beta$ & 1 & 0.2757 \\
\hline IGF1 & Insulin-like growth factor 1 (somatomedin C) & 14 & $<0.0001$ \\
\hline MAPK10 & Mitogen-activated protein kinase 10 & 3 & 0.0001 \\
\hline RAF1 & V-raf-1 murine leukemia viral oncogene homolog 1 & 1 & 0.4154 \\
\hline SGK1 & Serum/glucocorticoid regulated kinase 1 & 2 & 0.0173 \\
\hline TSC1 & Tuberous sclerosis 1 & 1 & 0.1861 \\
\hline BCL2L11 & BCL2-like 11 (apoptosis facilitator) & 4 & 0.0002 \\
\hline TLR2 & Toll-like receptor 2 & 1 & 0.5613 \\
\hline
\end{tabular}

viral transcription, the viral infectious cycle, viral reproduction and the bacterial invasion of epithelial cells. A previous clinical investigation revealed that the risk of bacterial and viral infections was seven times higher in MM patients compared with matched controls due to MM-associated immunodeficiency resulting from $\mathrm{PC}$ disorders, including $\mathrm{B}$-cell dysfunction, and T-cell, dendritic cell and NK cell abnormalities (45).
In conclusion, the present study identified and systematically analyzed predicted MM-associated target genes of miR-19a. A total of 121 differentially expressed genes in MM were identified, including 80 upregulated genes and 41 downregulated genes. Among these differentially expressed genes, RHOB, CLTC, PSAP and PPP6R2, were predicted target genes of miR-19a. The results of NLP analysis revealed that 


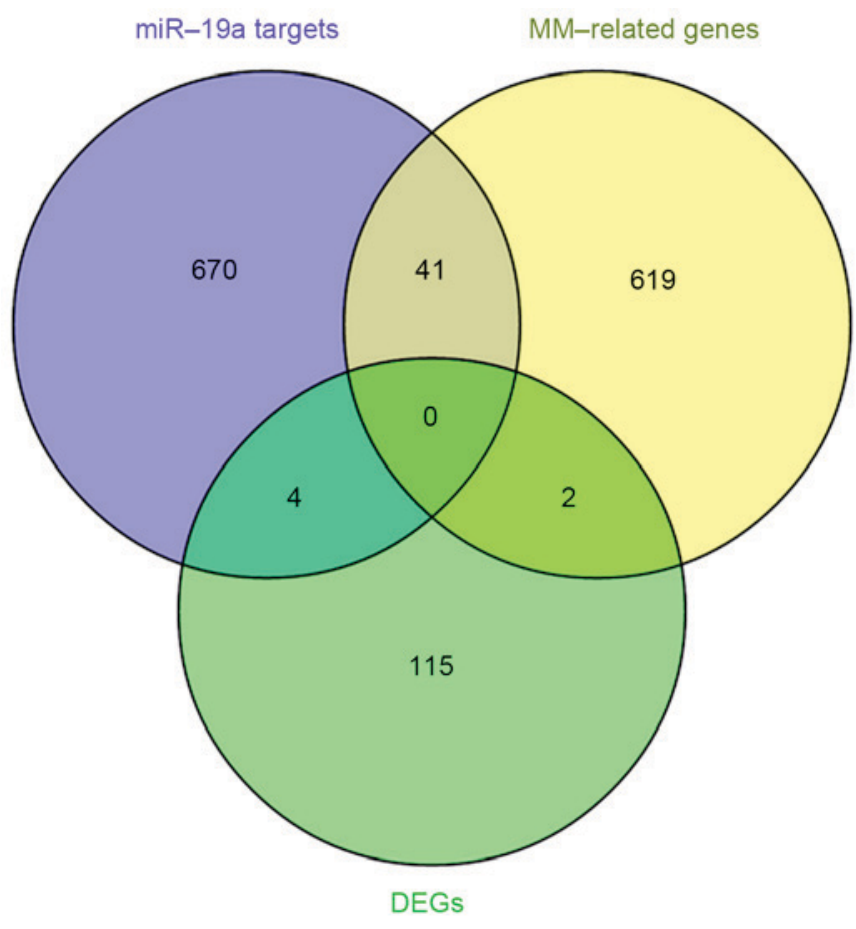

Figure 4. Overlap of the predicted target genes of miR-19a, DEGs in MM and MM-associated genes identified in natural language processing analysis miR, microRNA; DEGs, differentially expressed genes; MM, multiple myeloma.

2 of the differentially expressed genes, YBX1 and TRIAP1, were associated with MM. In addition, 41 target genes of miR-19a were associated with the development and progression of MM. The combined examination of gene expression and bioinformatical prediction for miR-19a target genes may provide new insights into carcinogenic mechanisms of MM, in addition to highlighting potential areas for the development of novel personalized therapies. Further studies are required to confirm the results of the present study in patients with MM.

\section{References}

1. Bommert K, Bargou RC and Stühmer T: Signalling and survival pathways in multiple myeloma. Eur J Cancer 42: 1574-1580, 2006 .

2. Benetatos L and Vartholomatos G: Deregulated microRNAs in multiple myeloma. Cancer 118: 878-887, 2012.

3. Raab MS, Podar K, Breitkreutz I, Richardson PG, Anderson KC: Multiple myeloma. Lancet 374: 324-339, 2009.

4. Dolloff NG and Talamo G: Targeted therapy of multiple myeloma. Adv Exp Med Biol 779: 197-221, 2013.

5. Chi J, Ballabio E, Chen XH, Kušec R, Taylor S, Hay D, Tramonti D, Saunders NJ, Littlewood T, Pezzella F, et al: MicroRNA expression in multiple myeloma is associated with genetic subtype, isotype and survival. Biol Direct 6: 23, 2011.

6. Carthew RW: Gene regulation by microRNAs. Curr Opin Genet Dev 16: 203-208, 2006.

7. Rushworth SA, Murray MY, Barrera LN, Heasman SA, Zaitseva L and Macewan DJ: Understanding the role of miRNA in regulating NF-kB in blood cancer. Am J Cancer Res 2: 65-74, 2012 .

8. Zhou Y, Chen L, Barlogie B, Stephens O, Wu X, Williams DR, Cartron MA, van Rhee F, Nair B, Waheed S, et al: High-risk myeloma is associated with global elevation of miRNAs and overexpression of EIF2C2/AGO2. Proc Natl Acad Sci USA 107: 7904-7909, 2010.
9. Lionetti M, Biasiolo M, Agnelli L, Todoerti K, Mosca L, Fabris S, Sales G, Deliliers GL, Bicciato S, Lombardi L, et al: Identification of microRNA expression patterns and definition of a microRNA/mRNA regulatory network in distinct molecular groups of multiple myeloma. Blood 114: e20-e26, 2009.

10. Wu P, Agnelli L, Walker BA, Todoerti K, Lionetti M, Johnson DC, Kaiser M, Mirabella F, Wardell C, Gregory WM, et al: Improved risk stratification in myeloma using a microRNA-based classifier. Br J Haematol 162: 348-359, 2013.

11. Mendell JT: miRiad roles for the miR-17-92 cluster in development and disease. Cell 133: 217-222, 2008.

12. Fuziwara CS and Kimura ET: Insights into regulation of the miR-17-92 cluster of miRNAs in cancer. Front Med (Lausanne) 2: 64, 2015.

13. Todoerti K, Barbui V, Pedrini O, Lionetti M, Fossati G, Mascagni P, Rambaldi A, Neri A, Introna M, Lombardi L and Golay J: Pleiotropic anti-myeloma activity of ITF2357: Inhibition of interleukin-6 receptor signaling and repression of miR-19a and miR-19b. Haematologica 95: 260-269, 2010.

14. Pichiorri F, Suh SS, Ladetto M, Kuehl M, Palumbo T, Drandi D, Taccioli C, Zanesi N, Alder H, Hagan JP, et al: MicroRNAs regulate critical genes associated with multiple myeloma pathogenesis. Proc Natl Acad Sci USA 105: 12885-12890, 2008.

15. Corthals SL, Sun SM, Kuiper R, de Knegt Y, Broyl A, van der Holt B, Beverloo HB, Peeters JK, el Jarari L, Lokhorst HM, et al: MicroRNA signatures characterize multiple myeloma patients. Leukemia 25: 1784-1789, 2011.

16. Chen L, Li C, Zhang R, Gao X, Qu X, Zhao M, Qiao C, Xu J and Li J: miR-17-92 cluster microRNAs confers tumorigenicity in multiple myeloma. Cancer Lett 309: 62-70, 2011.

17. Gutierrez NC, Sarasquete ME, Misiewicz-Krzeminska I, Delgado M, De Las Rivas J, Ticona FV, Fermiñán E, Martín-Jiménez P, Chillón C, Risueño A, et al: Deregulation of microRNA expression in the different genetic subtypes of multiple myeloma and correlation with gene expression profiling. Leukemia 24: 629-637, 2010.

18. Claudio JO, Masih-Khan E, Tang H, Goncalves J, Voralia M, Li ZH, Nadeem V, Cukerman E, Francisco-Pabalan O, Liew CC, et al: A molecular compendium of genes expressed in multiple myeloma. Blood 100: 2175-2186, 2002.

19. Dweep H, Sticht C, Pandey P and Gretz N: miRWalk-database: Prediction of possible miRNA binding sites by 'walking' the genes of three genomes. J Biomed Inform 44: 839-847, 2011.

20. Zhang F, Shi Y, Wang L and Sriram S: Role of HDAC3 on p53 expression and apoptosis in $\mathrm{T}$ cells of patients with multiple sclerosis. PLoS One 6: e16795, 2011.

21. Kemppinen AK, Kaprio J, Palotie A and Saarela J: Systematic review of genome-wide expression studies in multiple sclerosis. BMJ Open 1: e000053, 2011.

22. Gandhi KS, McKay FC, Cox M, Riveros C, Armstrong N, Heard RN, Vucic S, Williams DW, Stankovich J, Brown M, et al: The multiple sclerosis whole blood mRNA transcriptome and genetic associations indicate dysregulation of specific $\mathrm{T}$ cell pathways in pathogenesis. Hum Mol Genet 19: 2134-2143, 2010.

23. Ritchie ME, Phipson B, Wu D, Hu Y, Law CW, Shi W and Smyth GK: limma powers differential expression analyses for RNA-sequencing and microarray studies. Nucleic Acids Res 43: e47, 2015 .

24. Giot L, Bader JS, Brouwer C, Chaudhuri A, Kuang B, Li Y, Hao YL, Ooi CE, Godwin B, Vitols E, et al: A protein interaction map of Drosophila melanogaster. Science 302: 1727-1736, 2003.

25. Shannon P, Markiel A, Ozier O, Baliga NS, Wang JT, Ramage D, Amin N, Schwikowski B and Ideker T: Cytoscape: A software environment for integrated models of biomolecular interaction networks. Genome Res 13: 2498-2504, 2003.

26. Hoang B, Zhu L, Shi Y, Frost P, Yan H, Sharma S, Sharma S, Goodglick L, Dubinett S and Lichtenstein A: Oncogenic RAS mutations in myeloma cells selectively induce cox- 2 expression, which participates in enhanced adhesion to fibronectin and chemoresistance. Blood 107: 4484-4490, 2006

27. Steinbrunn T, Stühmer T, Gattenlöhner S, Rosenwald A, Mottok A, Unzicker C, Einsele H, Chatterjee M and Bargou RC: Mutated RAS and constitutively activated Akt delineate distinct oncogenic pathways, which independently contribute to multiple myeloma cell survival. Blood 117: 1998-2004, 2011.

28. Rasmussen T, Kuehl M, Lodahl M, Johnsen HE and Dahl IM: Possible roles for activating RAS mutations in the MGUS to MM transition and in the intramedullary to extramedullary transition in some plasma cell tumors. Blood 105: 317-323, 2005. 
29. Chng WJ, Gonzalez-Paz N, Price-Troska T, Jacobus S Rajkumar SV, Oken MM, Kyle RA, Henderson KJ, Van Wier S, Greipp P, et al: Clinical and biological significance of RAS mutations in multiple myeloma. Leukemia 22: 2280-2284, 2008.

30. Eliseeva IA, Kim ER, Guryanov SG, Ovchinnikov LP and Lyabin DN: Y-box-binding protein 1 (YB-1) and its functions. Biochemistry (Mosc) 76: 1402-1433, 2011.

31. Chatterjee M, Rancso C, Stuhmer T, Eckstein N, Andrulis M, Gerecke C, Lorentz H, Royer HD and Bargou RC: The Y-box binding protein $\mathrm{YB}-1$ is associated with progressive disease and mediates survival and drug resistance in multiple myeloma. Blood 111: 3714-3722, 2008.

32. Vaiman AV, Stromskaya TP, Rybalkina EY, Sorokin AV, Ovchinnikov LP and Stavrovskaya AA: Development of drug resistance in the population of colon cancer cells under the effect of multifunctional protein YB-1. Bull Exp Biol Med 143. 463-466, 2007.

33. Lasham A, Print CG, Woolley AG, Dunn SE and Braithwaite AW: YB-1: Oncoprotein, prognostic marker and therapeutic target? Biochem J 449: 11-23, 2013.

34. Zhou J, Zhu Y, Zhang G, Liu N, Sun L, Liu M, Qiu M, Luo D, Tang Q, Liao Z, et al: A distinct role of RhoB in gastric cancer suppression. Int J Cancer 128: 1057-1068, 2011.

35. Kim DM, Chung KS, Choi SJ, Jung YJ, Park SK, Han GH, Ha JS, Song KB, Choi NS, Kim HM, et al: RhoB induces apoptosis via direct interaction with TNFAIP1 in HeLa cells. Int J Cancer 125 2520-2527, 2009.

36. Tan Y, Yin H, Zhang H, Fang J, Zheng W, Li D, Li Y, Cao W, Sun C, Liang Y, et al: Sp1-driven up-regulation of miR-19a decreases RHOB and promotes pancreatic cancer. Oncotarget 6 : 17391-17403, 2015.

37. Chikatsu N, Kojima H, Suzukawa K, Shinagawa A, Nagasawa T, Ozawa H, Yamashita Y and Mori N: $\mathrm{ALK}^{+}, \mathrm{CD}^{-} 0^{-} \mathrm{CD}^{-} 0^{-}$large B-cell lymphoma containing anaplastic lymphoma kinase (ALK) fused to clathrin heavy chain gene (CLTC). Mod Pathol 16 828-832, 2003.
38. Cerchietti L, Damm-Welk C, Vater I, Klapper W, Harder L, Pott C, Yang SN, Reiter A, Siebert R, Melnick A and Woessmann W: Inhibition of anaplastic lymphoma kinase (ALK) activity provides a therapeutic approach for CLTC-ALK-positive human diffuse large B cell lymphomas. PLoS One 6: e18436, 2011.

39. Gunia S, Koch S, May M,Dietel M and Erbersdobler A: Expression of prostatic acid phosphatase (PSAP) in transurethral resection specimens of the prostate is predictive of histopathologic tumor stage in subsequent radical prostatectomies. Virchows Arch 454: 573-579, 2009.

40. Pawar H, Kashyap MK, Sahasrabuddhe NA, Renuse S, Harsha HC, Kumar P, Sharma J, Kandasamy K, Marimuthu A, Nair B, et al: Quantitative tissue proteomics of esophageal squamous cell carcinoma for novel biomarker discovery. Cancer Biol Ther 12: 510-522, 2011.

41. Kato JY and Yoneda-Kato N: Mammalian COP9 signalosome. Genes Cells 14: 1209-1225, 2009.

42. Schütz AK, Hennes T, Jumpertz S, Fuchs S and Bernhagen J: Role of CSN5/JAB1 in Wnt/ $\beta$-catenin activation in colorectal cancer cells. FEBS Lett 586: 1645-1651, 2012.

43. Mori M, Yoneda-Kato N, Yoshida A and Kato JY: Stable form of JAB1 enhances proliferation and maintenance of hematopoietic progenitors. J Biol Chem 283: 29011-29021, 2008.

44. Tsujimoto I, Yoshida A, Yoneda-Kato N and Kato JY: Depletion of CSN5 inhibits Ras-mediated tumorigenesis by inducing premature senescence in p53-null cells. FEBS Lett 586: 4326-4331, 2012

45. Blimark C,Holmberg E,Mellqvist UH,Landgren O,Björkholm M, Hultcrantz M, Kjellander C, Turesson I and Kristinsson SY: Multiple myeloma and infections: A population-based study on 9253 multiple myeloma patients. Haematologica 100: 107-113, 2015. 